International Journal of Pure and Applied Mathematics

Volume 82 No. 4 2013, 531-546

ISSN: 1311-8080 (printed version); ISSN: 1314-3395 (on-line version)

url: http://www.ijpam.eu

doi: http://dx.doi.org/10.12732/ijpam.v82i4.3

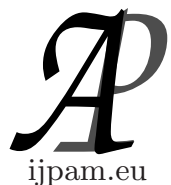

\title{
GRAPH AND GOLDBACH CONJECTURE
}

\author{
Bichitra Kalita \\ Department of Computer Application (M.C.A) \\ Assam Engineering College \\ Guwahati, 781013, Assam, INDIA
}

\begin{abstract}
In this paper, the new direction of proof of Gold Bach conjecture "every even number n greater than 2 can be expressed as a sum of two primes" has been forwarded with the help of some graph theoretic concept which seems to be very easy. This new direction gives the application graph theory in proving the conjectural problem Gold Bach conjecture of number theory. Some theoretical results have also been focused.
\end{abstract}

AMS Subject Classification: 11A41, 11A51, 11A051, 05C30, 05C45 Key Words: the graph PVEEWG, BKSTPVEEWG, CPVEEWG, EEWE

\section{Introduction}

We generally consider the number theory as branch of pure mathematics and graph theory as a branch of an applied due it's application in different fields. It has been found that the partition functions give some idea of graphical partitions of even numbers. But, no one new finding has been found for deep relationship between numbers and graphs. The present article has been considered to know the current trends of graph theory with number theory, which is assumed to be a new trends. We know that the Number Theory plays a very important role in the branch of Pure Mathematics. It has been occupying a

Received: June 23, 2012

(c) 2013 Academic Publications, Ltd. url: www.acadpubl.eu 
field of so many conjectural problems or some conjectural statements since the discovery of number theory. It can be considered as a never ending theory as and when any one problem of number theory, of course conjectural problem, is solved, immediately there may exist another new problem, which is also assumed to be conjectural. There are so many theorems / results relating to prime numbers, quite a few of them are fundamental theorem of arithmetic, Fermat's little theorem, Wilson's theorem, perfect number conjectures, prime number theorem, Goldbach conjecture, Twin prime conjectures etc. It has been found that $2^{\mathrm{p}-1}\left(2^{\mathrm{p}}-1\right)$ is a perfect number, where $2^{\mathrm{p}}-1$ is a prime for any prime $\mathrm{p}$. It has also been found that there is no general formula to know the consecutive primes $\geq 13$. The formula $m^{2}-m+41$ gave forty primes for $1 \leq \mathrm{m} \leq 40$ [1], but they were not consecutive. Again, another formula $\mathrm{m}^{2}-79 \mathrm{~m}+1601$ has been found [2] which gives 79 primes for $1 \leq \mathrm{m} \leq 79$, but it fails for $\mathrm{m}=80$. A direction of proof of Goldbach conjecture has been forwarded by Kalita [3] in 2000. Thereafter, a theoretical proof of Goldbah conjecture has been forwarded by Kalita [4] in 2006 in opposite direction. That is, every sum of two primes gives all even numbers. Alex Vand etal [5] showed that the Goldbach conjecture is true for all the even numbers from 2 to $10^{18}$ with a comment after the publication of proof of Goldbach conjecture forwarded by Kalita. A theorem relating to the existence of consecutive primes $\geq 13$ has also been forwarded by Kalita. Two new conjectures have also been proposed for existence and non- existence of perfect numbers. He considered all the even numbers by two sets such that $\{4 n+10 / n \geq 4\}$ and $\{4 n+12 / n \geq 4\}$, whose union gives all even numbers $\geq 26$. Some additional properties of the sets $\{4 n+10 / n \geq 4\}$ and $\{4 n+12 / n \geq 4\}$ have also been discussed by Kalita [6] in 2009. The theoretical explanations relating to pentagonal numbers, partition of numbers, graphical partition and perfect numbers have been discussed from the two sets of even numbers $\{4 n+10 / n \geq 4\}$ and $\{4 n+12 / n \geq 4\}$. Some properties of even numbers with various properties of graphs have also been discussed by Kalita.etal [7] in 2009. An algorithm has also been forwarded to know the various forms /structures of graphs relating to the even numbers $2 n+4,4 n+4,(n+1)(n+2)$ and $6 \mathrm{n}+2$ for $\mathrm{n} \geq 1$.

In this paper, the proof of Goldbach conjecture "Every even number $\mathrm{n}>2$ can be expressed as a sum of two primes" has been forwarded with the help of some graph theoretic concept. This will give a new direction of application of graph theory for the proof of long standing Gold Bach conjecture. Various properties of graph relating to the prime numbers have also been focused.

The paper is organized as follows: We just have focused some previous works of number theory and a few works of graph theory in section1.0. In section 2.0, 
we consider some known definition of graph and use some terminology relating to graph. Some new definitions are considered in this section. Some theorems are established in section 3.0. Section 4.0 is included for the proof of Goldbach conjecture. The conclusion is included in section 5.0.

\section{Definition and Terminology}

Before going to forward the proof of Goldbach conjecture, statement of which is known as every even number $n$ larger than 2 can be expressed as a sum of two primes, we remind some definitions of graph (not all of course) and consider some terminologies. Thereafter, we shall supply Some new definitions, which will help in proving the Goldbach conjecture.

2.1. Graph A graph $G(V, E)$ consists of a finite non-empty set V (called set of vertices) together with a set $\mathrm{E}$ (called the set of edges) of unordered pair of distinct points of $\mathrm{V}$. The graph of four vertices and five edges is shown in figure-1.

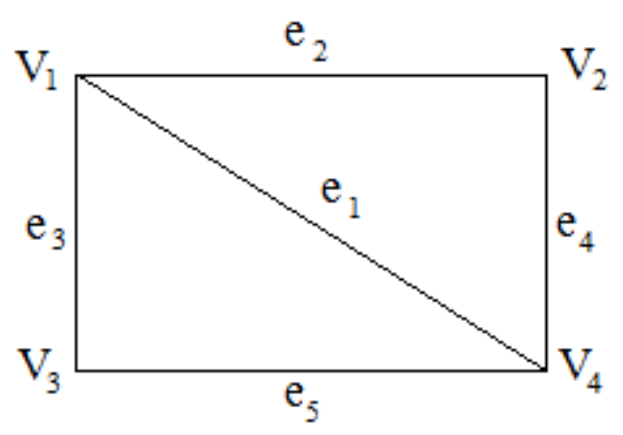

Figure 1

Here, for the edge say $\mathrm{e}_{2}=\left(\mathrm{v}_{1}, \mathrm{v}_{2}\right)$, the vertices $\mathrm{v}_{1}$ and $\mathrm{v}_{2}$ are called end vertices of the edge $\mathrm{e}_{2}$. Again the edge $\mathrm{e}_{2}$ is called incident with $\mathrm{v}_{1}$ and $\mathrm{v}_{2}$.

2.2. Degree of a Vertex The number of edges incident with the vertex with self loop counted twice is called the degree of that vertex. A vertex of a graph is called a pendent vertex if the degree of the vertex is one.

Note 1. The graph of figure -1 has no self loop and parallel edges. We only shall consider the graph having only self loops but not parallel edges, as our discussion does not have any effect in case of graph with parallel edges. 
2.3. Complete Graph. A graph $\mathrm{G}(\mathrm{V}, \mathrm{E})$ is called complete if there is an edge between every pair of vertices. The complete graph of $n$ vertices is denoted by $K_{n}$ and it has $n(n-1) / 2$ number of edges.

Definitions 2.4. Prime Vertex and Even Edge Weighted Graph (PVEEWG): Let G $(\mathrm{V}, \mathrm{E})$ be a graph having at least one self loop without parallel edges. For the graph $G(V, E)$ we consider the set of vertices $V=\left\{V_{1}\right.$, $\left.\mathrm{V}_{2}, \mathrm{~V}_{3}, \ldots \mathrm{V}_{\mathrm{n}}\right\}$ and the set of edges $\mathrm{E}=\left\{\mathrm{E}_{1}, \mathrm{E}_{2}, \mathrm{E}_{3}, \ldots \mathrm{E}_{\mathrm{n}}\right\}$. Let $\mathrm{P}_{1}<\mathrm{P}_{2}<\mathrm{P}_{3}$ $<\mathrm{P}_{4} \ldots<\mathrm{P}_{\mathrm{n}}$ for $\mathrm{n} \geq 5$ be consecutive primes $\geq 13$. Now attaching these primes $\mathrm{P}_{1}<\mathrm{P}_{2}<\mathrm{P}_{3}<\mathrm{P}_{4} \ldots .<\mathrm{P}_{\mathrm{n}}$ for $\mathrm{n} \geq 5$ with the vertices $\mathrm{V}_{1}, \mathrm{~V}_{2}, \mathrm{~V}_{3}, \ldots$ Vn of the graph $\mathrm{G}$ which are now called prime weight $(\mathrm{PW})$ of the vertices and considering weights $\mathrm{W}_{1}, \mathrm{~W}_{2}, \mathrm{~W}_{3}, \ldots \ldots \mathrm{W}_{\mathrm{n}}$ for the edges $\mathrm{E}_{1}, \mathrm{E}_{2}, \mathrm{E}_{3} \ldots \mathrm{E}_{\mathrm{n}}$, where the weights $\mathrm{W}_{1}, \mathrm{~W}_{2}, \mathrm{~W}_{3}, \ldots . . \mathrm{W}_{\mathrm{n}}$ are obtained as a sum of any two prime weights $\mathrm{P}_{\mathrm{i}}+$ $\mathrm{P}_{\mathrm{j}}$ of attached vertices and considering the self loop, if exists in the graph as sum of the same prime weight of the attached vertex, we can construct a new graph with prime vertex set $\mathrm{V}_{\mathrm{atP}}$ and the weighted edges set $\mathrm{E}_{\mathrm{soP}}$ and this graph, thus obtained is called a prime vertex and even edge weighted graph of the graph $\mathrm{G}(\mathrm{V}, \mathrm{E})$ and it is denoted by the graph PVEEWG ( $\mathbf{V}$ atP, $\left.\mathbf{E}_{\mathbf{s o P}}\right)$. [ The notation of the graph PVEEWG ( $\left.\mathbf{V}_{\text {atP }}, \mathbf{E}_{\mathbf{s o P}}\right)$ includes prime weights for the vertices [means prime numbers] and the even edge weights of the edges are always even number]. The following graph (figure-2) is a prime vertex and even edge weighted graph PVEEWG $\left(\mathbf{V}_{\mathbf{a t P}}, \mathbf{E}_{\mathbf{s o}}\right.$ ) for the five primes $13<17$ $<19<23<29$. For this graph $\mathrm{V}_{\mathrm{atP}}=\{13,17,19,23,29\}$ and Esop $=\left\{\mathrm{W}_{1}=30, \mathrm{~W}_{2}\right.$ $\left.=34, \mathrm{~W}_{3}=36, \mathrm{~W}_{4}=40, \mathrm{~W}_{5}=42, \mathrm{~W}_{6}=42, \mathrm{~W}_{7}=52\right\}$ and the original graph (Figure-2) has one self loop and this gives an edge of weight $\mathrm{W}_{2}=34$.

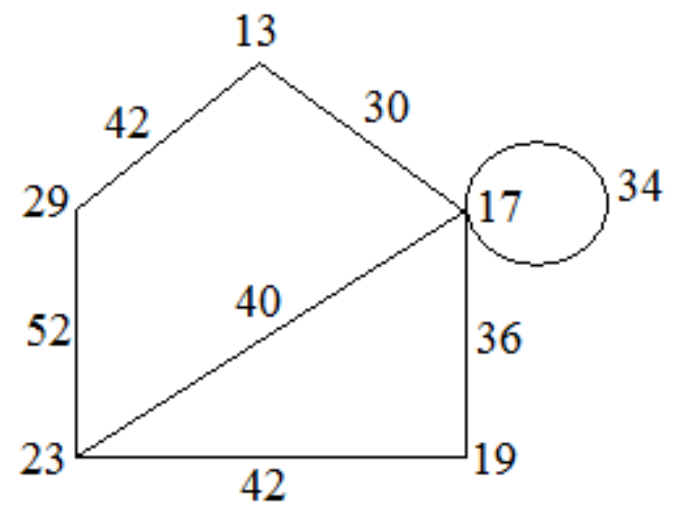

Figure 2 
Note 2. When we get the graph PVEEWG ( $\mathbf{V}$ atP, $\left.\mathbf{E}_{\text {soP }}\right)$ we do not take or do not consider this graph as a label graph though it seems to be label graph. We only to use this graph for the proof of Gold Bach conjecture which will be discussed later, but not for all other properties of label graph.

Note 3. The self loop indicate the sum of same prime weight. The sum of prime weight $17+17$ equal 34 and this is a self loop of weight 34 (figure-2 above).

Note 4. We do not consider the properties of label graph such as the number of simple labeled graphs of $\mathrm{n}$ vertices is $2^{\mathrm{n}(\mathrm{n}-1) / 2}$ as our graph is not simple which contains at least one self loop.

Note 5. One can construct the graph PVEEWG $\left(\mathbf{V}_{\text {atP }}, \mathbf{E}_{\mathbf{s o P}}\right)$ for the primes $\mathrm{P}_{1} \quad \leq \mathrm{P}_{2} \quad \leq \mathrm{P}_{3} \quad \leq \ldots \leq \mathrm{P}_{\mathrm{n}}$ for the same graph changing the attachment of prime weights with the vertices $V_{1}, V_{2}, V_{3}, \ldots V_{n}$ with the primes. The changed pattern of the attachment of prime weights are as follows:

$\mathrm{V}_{1} \rightarrow \mathrm{P}_{1}, \mathrm{~V}_{2} \rightarrow \mathrm{P} 2, \ldots \mathrm{V}_{\mathrm{n}} \rightarrow \mathrm{P}_{\mathrm{n}}$,

$\mathrm{V}_{1} \rightarrow \mathrm{P}_{2}, \mathrm{~V}_{2} \rightarrow \mathrm{P}_{3} \ldots \mathrm{Vn} \rightarrow \mathrm{P}_{1}$,

$\mathrm{V}_{1} \rightarrow \mathrm{P}_{3}, \mathrm{~V}_{2} \rightarrow \mathrm{P}_{4} \ldots \mathrm{V}_{\mathrm{n}} \rightarrow \mathrm{P}_{2}, \ldots \mathrm{V}_{1} \rightarrow \mathrm{P}_{\mathrm{n}}, \mathrm{V}_{2} \rightarrow \mathrm{P}_{\mathrm{n}+1}, \ldots \mathrm{V}_{\mathrm{n}} \rightarrow \mathrm{P}_{1}$. These graphs also gives the different types of graph PVEEWG $\left(\mathbf{V}_{\text {atP }}, \mathbf{E}_{\mathbf{s o P}}\right)$ with different weights as defined above.

Note 6. In the graph PVEEWG ( $\left.\mathbf{V}_{\text {atP }}, \mathbf{E}_{\text {soP }}\right)$ same weighted edges may exist more than once. But they will be considered as different edges.

Properties 1. The even edge weights of the graph PVEEWG $\left(\mathbf{V}_{\text {atP }}, \mathbf{E}_{\mathbf{s o P}}\right)$ always represent

the even number.

2. The prime vertices of the graph PVEEWG $\left(\mathbf{V}_{\text {atP }}, \mathbf{E}_{\mathbf{s o P}}\right)$ always represent the

attachment of prime numbers with the vertices of the graph.

3. The number of vertices and edges of the graph PVEEWG $\left(\mathbf{V}_{\text {atP }}, \mathbf{E}_{\mathbf{s o P}}\right)$ is equal

to the number of vertices and edges of the original graph.

2.5. BK'S Special Type Prime Vertex and Even Edge Weighted graph (BKSTPVEEWG): Let $G(V, E)$ be a graph with self loops and pendent vertices. Then the graph PVEEWG $\left(\mathbf{V}_{\text {atP }}, \mathbf{E}_{\text {soP }}\right)$ obtained from the graph G (V,E) is called the BK'S Special Type Prime Vertex and Even Edge Weighted graph (BKSTPVEEWG) [Bichitra Kalita's special type prime vertex and even edge weighted graph] for the primes $\mathrm{P}_{1}<\mathrm{P}_{2}<\mathrm{P}_{3}<\mathrm{P}_{4}$ 
$<\mathrm{P}_{5} \ldots<\mathrm{P}_{\mathrm{n}}$ for $\mathrm{n} \geq 5$ consecutive primes where there are attachment of some prime weights with the pendent vertices of the graph many times, satisfying the following conditions:

i) It must have $n-2$ self loops for $n \geq 5$ consecutive primes, which means that there are n-2 weighted edges which are obtained from the sum of same prime weight.

ii) The degrees of the vertices of the graph BKSTPVEEWG are always expressed as an n-tupple forms such that (n-1,n+1,n,n-1,n-4), (n-1,n+1,n,n-1,n$2, \mathrm{n}-5),(\mathrm{n}-1, \mathrm{n}+1, \mathrm{n}, \mathrm{n}-1, \mathrm{n}-2, \mathrm{n}-3, \mathrm{n}-6), \ldots$ for simultaneous changes of $\mathrm{n} \geq 5$ for the primes $\mathrm{P}_{1}<\mathrm{P}_{2}<\mathrm{P}_{3}<\mathrm{P}_{4} \ldots<\mathrm{P}_{\mathrm{n}}$.

iii) There are $\mathrm{N}-(\mathrm{n}-1)$ number of pendent vertices where $\mathrm{N}$ is the number of vertices of the graph obtained after the attachment of prime vertices and this is equal with the vertex set $\mathrm{V}$ of the graph. This graph is denoted by the graph BKSTPVEEWG $\left(V_{\mathrm{atP}}, \mathrm{E}_{\mathrm{soP}}\right)$, where $\mathrm{V}_{\mathrm{atP}}$ is the set of vertices, which are same with the original graph after attachment of some prime vertices many times with some vertices of the graph and there are $5 \mathrm{i}+6$ number of vertices for $\mathrm{i} \geq 1$ for simultaneous changes of $\mathrm{n} \geq 5$ and $\mathrm{E}_{\mathrm{soP}}$ is the set of even weighted edges as defined in graph PVEEWG ( $\left.\mathrm{V}_{\text {atP }}, \mathrm{E}_{\mathrm{soP}}\right)$ above [2.4]

The Figure-3 is a graph BKSTPVEEWG $\left(\mathrm{V}_{\mathrm{atP}}, \mathrm{E}_{\mathrm{soP}}\right)$, for the primes 13, 17, 19, 23 and 29.

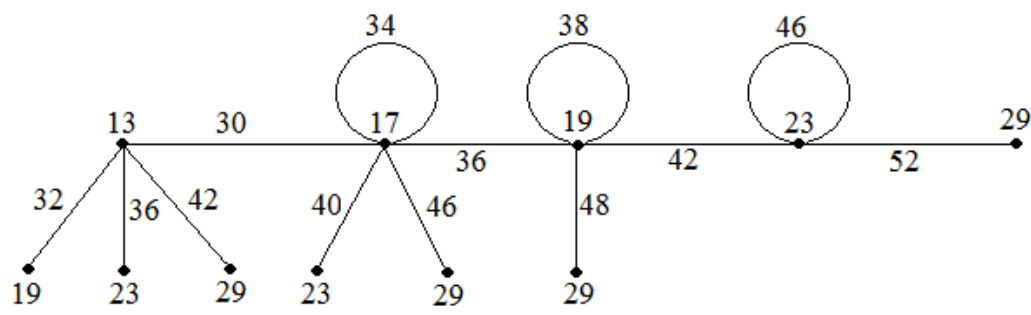

Figure 3

Here (i) the number of self loop is $\mathrm{n}-2=5-2=3$, which gives three even weighted edges 34,38 and 46 .[ figure 3 -above]

(ii) The degrees of the vertices are 5 -tupple form for $n=5$.

That is $(\mathrm{n}-1, \mathrm{n}+1, \mathrm{n}, \mathrm{n}-1, \mathrm{n}-4)=(4,6,5,4,1)$, which means that there are two vertices of degree four, one vertex of degree six, one vertex of degree five and seven vertex of degree one.

(iii) There are $\mathrm{N}-(\mathrm{n}-1)=11-(5-1)=7$ number of pendent vertices, and $\mathrm{V}_{\mathrm{atP}}=\{13,17,19,23,29,19,23,29,23,29,29\}$ and $\mathrm{E}_{\mathrm{soP}}=\{30,32,34,36,36,38,40$, 


\section{$42,42,46,46,48,52\}$}

2.6. Complete Prime Vertex and Even edge weighted Graph: A graph PVEEWG ( $\mathbf{V}$ atP, $\left.\mathbf{E}_{\mathbf{s o P}}\right)$ is called a complete prime vertex and even edge weighted graph if it is obtained from a complete graph $G(V, E)$. It is denoted by the graph CPVEEWG ( $\left.\mathbf{V}_{\mathbf{a t P}}, \mathbf{E}_{\mathbf{S o P}}\right)$. According to the definition of complete graph of $n$ vertices there are $n(n-1) / 2$ number of edges, which is the known result of graph theory. Hence, in the graph CPVEEWG $\left(\mathbf{V}_{\mathbf{a t P}}, \mathbf{E}_{\mathbf{s o P}}\right.$ ), there are also $n(n-1) / 2$ number of even weighted edges (even numbers). The figure- 4 [discussed later] is a complete prime vertex even edge weighted graph.

\section{Theorems and Results}

The following theorems are important for the proof of Golbach conjecture.

Theorem 3.1. Let $\mathrm{P}_{1}<\mathrm{P}_{2}<\mathrm{P}_{3}<\mathrm{P}_{4} \ldots<\mathrm{P}_{\mathrm{n}}$ be consecutive primes $\geq 13$ and $\mathrm{n} \geq 5$. Then there exist a graph $\mathbf{C P V E E W G}\left(\mathbf{V}_{\mathbf{a t P}}, \mathbf{E}_{\mathbf{s o P}}\right)$ for every complete graph $\mathrm{G}(\mathrm{V}, \mathrm{E})$ and there are $\mathrm{n}(\mathrm{n}-1) / 2$ number of even weighted edges.

Proof. We supply the proof of the theorem considering some complete prime vertex and even weighted edges graphs. Let $n=5$. Hence there are five consecutive primes $13<17<19<23<29$. Now we can construct a graph CPVEEWG $\left(\mathbf{V}_{\text {atP }}, \mathbf{E}_{\text {soP }}\right)$ with five prime vertices attaching prime numbers $13,17,19,23$ and 29 with the vertices of the complete graph as defined in section 2.6 which is shown in figure- 4 and there are $n(n-1) / 2=10$ even weighted edges and this is true from graph theory (From definition of complete graph). These even weighted edges are $\mathrm{w}_{1}=30, \mathrm{w}_{2}=32, \mathrm{w}_{3}=36, \mathrm{w}_{4}=36, \mathrm{w}_{5}=40$, $\mathrm{w}_{6}=42, \mathrm{w}_{7}=42, \mathrm{w}_{8}=46, \mathrm{w}_{9}=48, \mathrm{w}_{10}=52$.

Again if we suppose $n=6$ and then we have six consecutive prime numbers 13, 17, 19, 23, 29, 31 and we can construct another graph CPVEEWG $\left(\mathbf{V}_{\text {atP }}, \mathbf{E}_{\text {soP }}\right)$ with six prime vertices as discussed above and there are $\mathrm{n}(\mathrm{n}$ 1) $/ 2=15$ even weighted edges and these weighted edges are $\mathrm{w}_{1}=30, \mathrm{w}_{2}=32$, $\mathrm{w}_{3}=36, \mathrm{w}_{4}=42, \mathrm{w}_{5}=44, \mathrm{w}_{6}=48, \mathrm{w}_{7}=46, \mathrm{w}_{8}=40, \mathrm{w}_{9}=36, \mathrm{w}_{10}=50$, $\mathrm{w}_{11}=48, \mathrm{w}_{12}=42, \mathrm{w}_{13}=54, \mathrm{w}_{14}=52$ and $\mathrm{w}_{15}=60$.

Continuing the process of construction of a graph CPVEEWG $\left(\mathbf{V}_{\text {atP }}, \mathbf{E}_{\mathbf{s o P}}\right)$ with $\mathrm{n}$ prime vertices attaching all the consecutive primes $\mathrm{P}_{1}<\mathrm{P}_{2}<\mathrm{P}_{3}<\mathrm{P}_{4}$ $<\mathrm{P}_{5} \ldots<\mathrm{P}_{\mathrm{n}}$ with the vertices of the complete graph $\mathrm{G}(\mathrm{V}, \mathrm{E})$ of $\mathrm{n}$ vertices and connecting every pair of vertices by the even weighted edges, as defined for the graph PVEEWG ( $\mathrm{V}$ atP, $\left.\mathrm{E}_{\mathrm{soP}}\right)$, we have there are $\mathrm{n}(\mathrm{n}-1) / 2$ number of even weighted edges, which is true from graph theory as a complete graph 


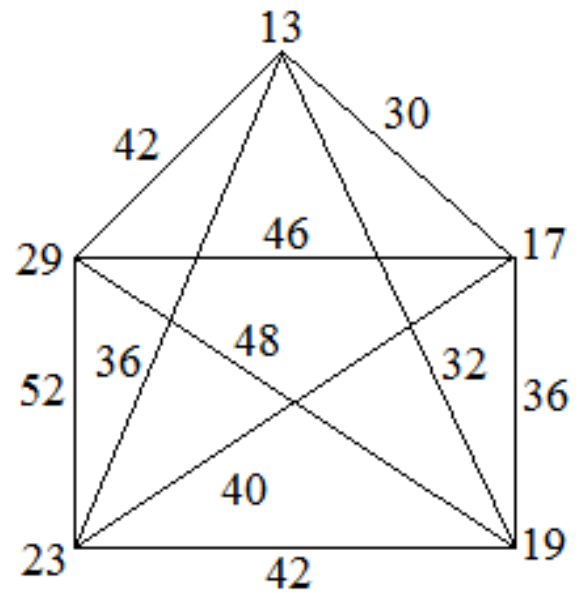

Figure 4

of $\mathrm{n}$ vertices always has $\mathrm{n}(\mathrm{n}-1) / 2$ edges. Hence there always exist a graph CPVEEWG ( $\left.\mathbf{V}_{\text {atP }}, \mathbf{E}_{\text {soP }}\right)$ for every complete graph of $n$ vertices. The existence of the graph CPVEEWG $\left(\mathbf{V}_{\text {atP }}, \mathbf{E}_{\mathbf{s o P}}\right)$ for $\mathrm{n} \geq 5$ consecutives prime vertices from the complete graph $G(V, E)$ of $n$ vertices and $n(n-1) / 2$ number of edges can also be shown from the following graph of CPVEEWG [ figure-5] of the graph CPVEEWG ( $\left.\mathbf{V}_{\mathbf{a t P}}, \mathbf{E}_{\text {soP }}\right)$. For the graph of CPVEEWG $\left(\mathrm{V}_{\mathrm{atP}}, \mathrm{E}_{\mathrm{soP}}\right)$, $\mathrm{x}$-axis represents the consecutive prime vertices and the values $\mathrm{y}$-axis represents the even weighted edges $=$ even numbers.

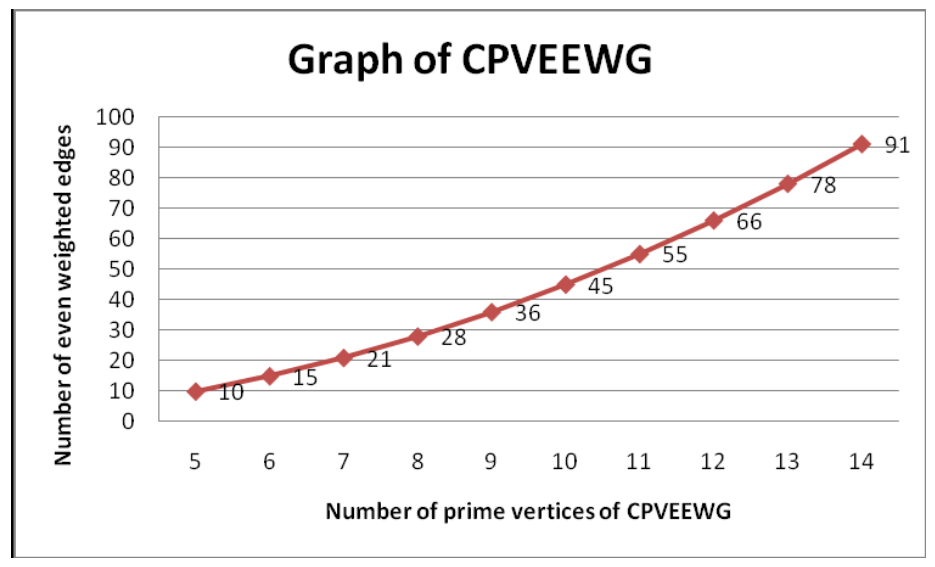

Figure 5 
Hence from the graph of figure- 5 it is cleared that the existence of even weighted edges $=$ even numbers increases to infinity when the number of prime vertices increases to infinity. The above graph [figure-5] gives only the existence of $10,15,21,28,36,45,55,66,78$ and 91 even numbers =even weighted edges for ten consecutive prime numbers separately. Hence from the graph above also we can have that there always exist a graph $\mathbf{C P V E E W G}\left(\mathbf{V}_{\mathbf{a t P}}, \mathbf{E}_{\mathbf{s o P}}\right)$ for $\mathrm{n} \geq 5$ consecutive prime vertices for the complete graph $\mathrm{G}(\mathrm{V}, \mathrm{E})$ of $\mathrm{n}$ vertices and there are $\mathrm{n}(\mathrm{n}-1) / 2$ number of even weighted edges.

Hence the proof.

Remark 1. From the above theorem 3.1 we have all the even numbers $n$ (n-1)/2 for $n \geq 5$, which are the even weighted edges of the graph CPVEEWG $\left(\mathbf{V}_{\text {atP }}, \mathbf{E}_{\mathbf{s o P}}\right)$ and they are actually obtained as a sum of two prime weights that is sum of two prime numbers of attached vertices of the complete graph. Hence we can comment that there are infinite number of even numbers which are the even weighted edges of the graph CPVEEWG $\left(\mathbf{V}_{\text {atP }}, \mathbf{E}_{\mathbf{s o}} \mathbf{P}\right)$ when $n$ tends to infinity. Hence, when the number of vertices tend to infinity for the complete graph then the number of even weighted edges of the graph CPVEEWG $\left(\mathbf{V}_{\text {atP }}, \mathbf{E}_{\mathbf{s o P}}\right)$ also tend to infinity, which means that there exist infinite number of even numbers which are obtained as a sum of two primes. But, we have one interesting open question for existence of consecutive even weighted edges $=$ even numbers which is discussed below

Open Question. Is the even weighted edges n(n-1)/2 of the graph CPVE EWG $\left(\mathbf{V}_{\text {atP }}, \mathbf{E}_{\text {soP }}\right)$ are consecutive? No. If they are consecutive, then our search for proof of Goldbach conjecture is finished here. But, they do not give consecutive even weighted edges. For example, if we consider the graph CPVEEWG ( $\mathbf{V}_{\text {atP }}, \mathbf{E}_{\text {soP }}$ ) for $n=5$ consecutive primes $13<17<19<23<29$ [Figfure-4 above] then we have ten even weighted edges or ten even numbers $\mathrm{w}_{1}=30, \mathrm{w}_{2}=32, \mathrm{w}_{3}=36, \mathrm{w}_{4}=36, \mathrm{w}_{5}=40, \mathrm{w}_{6}=42, \mathrm{w}_{7}=42, \mathrm{w}_{8}=46$, $\mathrm{w}_{9}=48, \mathrm{w}_{10}=52$. But they are not consecutive as the even numbers 34, 38, 44. 50 are not found from the graph $\mathbf{C P V E E W G}\left(\mathbf{V}_{\mathbf{a t P}}, \mathbf{E}_{\mathbf{s o P}}\right)$. Hence we must search the even weighted edges $=$ even numbers which are not obtained from the graph CPVEEWG $\left(\mathbf{V}_{\text {atP }}, \mathbf{E}_{\text {soP }}\right)$ and they will form a set $\mathbf{E E W E}$ (exceptional even weighted edges=even numbers) whose explanation is discussed in REMARK2.

Remark 2. (Exceptional Even Weighted Edge) The even weighted edges which lie in the graph BKSTPVEEWG $\left(\mathrm{V}_{\mathrm{atP}}, \mathrm{E}_{\mathrm{soP}}\right)$, but does not lie in the graph CPVEEWG $\left(\mathbf{V}_{\text {atP }}, \mathbf{E}_{\mathbf{s o P}}\right)$, due to which the number of edges $\mathrm{n}(\mathrm{n}$ 1)/2 of the graph CPVEEWG $\left(\mathbf{V}_{\mathbf{a t P}}, \mathbf{E}_{\mathbf{s o P}}\right)$ does not form the set of all 
consecutive even weighted edges $=$ even numbers are called exceptional even weighted edges, and the set of exceptional even weighted edges is denoted by EEWE.

Exception. The exceptional edges are not defined in graph theory, but, we shall consider exceptional even weighted edges only for the proof of Gold Bach conjecture. Hence this is the exception here.

Theorem 3.2 The elements the set EEWE can be obtained from the graph BKSTPVEEWG $\left(\mathrm{V}_{\mathrm{atP}}, \mathrm{E}_{\mathrm{soP}}\right)$ which are not found from the theorem3.1.

Proof. The theorem 3.1 clearly explains that one can construct a graph CPVEEWG ( $\mathbf{V}_{\text {atP }}, \mathbf{E}_{\text {soP }}$ ) from a complete graph $\mathrm{G}(\mathrm{V}, \mathrm{E})$ of $\mathrm{n}$ vertices with the consecutive prime vertices $\mathbf{P}_{\mathbf{1}}<\mathbf{P}_{\mathbf{2}}<\mathbf{P}_{\mathbf{3}}<\mathrm{P}_{4} \ldots<\mathrm{P}_{\mathrm{n}}$ for $\mathrm{n} \geq 5$ primes $\geq 13$ with $\mathrm{n}(\mathrm{n}-1) / 2$ number of even weighted edges. Besides, it is known that there are $n(n-1) / 2$ number of even weighted edges but they are not consecutive. The even weighted edges which are obtained from the graph CPVEEWG $\left(\mathbf{V}_{\text {atP }}, \mathbf{E}_{\mathbf{s o P}}\right)$ for $\mathrm{n}=5$ consecutive prime vertices are $30,32,36,36,40,42$, 42, 46, 48, 52.[discussed above]. But the exceptional even weighted edges $34,38,44,50$ are not found here due to which the set of edges of the graph CPVEEWG ( $\left.\mathbf{V}_{\text {atP }}, \mathbf{E}_{\text {soP }}\right)$ does not form the consecutive even weighted edges $=$ consecutive even numbers. Therefore, we now proceeds to find out the set $\mathbf{E E W E}=\{34,38,44,50\}$ from the graph BKSTPVEEWG $\left(\mathrm{V}_{\mathrm{atP}}, \mathrm{E}_{\mathrm{soP}}\right)$. Let us consider the different values of $n \geq 6$ consecutive primes and let us now construct a graph BKSTPVEEWG $\left(\mathrm{V}_{\text {atP }}, \mathrm{E}_{\mathrm{soP}}\right)$ for the prime vertices $\mathrm{P}_{1}$ $<\mathrm{P}_{2}<\mathrm{P}_{3}<\mathrm{P}_{4}<\mathrm{P}_{5}<\mathrm{P}_{6}$, that is for the prime vertices 13,17,19,23,29,31.(n=6) in which the elements of the set EEWE exist which are found from the following figure -6

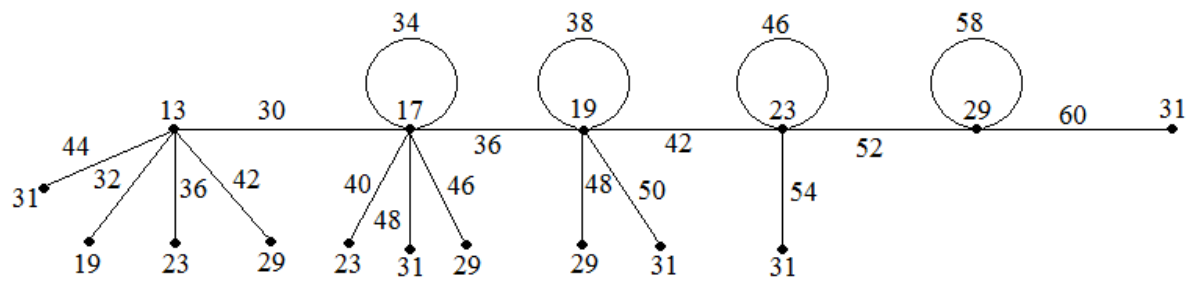

Figure 6

The graph BKSTPVEEWG $\left(\mathrm{V}_{\text {atP }}, \mathrm{E}_{\mathrm{soP}}\right)$ of Figure-6 above gives 19 even weighted edges which means that there are 19 even weighted edges with four repeated even weights and they are $30,32,34, \underline{36,36}, 38,40, \underline{42,42}, 44, \underline{46,46}$, 
$48,48,50,52,54,58$ and 60 . The even weighted edges 34, 38, 44 and 50 [even numbers] are found now from the graph BKSTPVEEWG $\left(\mathrm{V}_{\mathrm{atP}}, \mathrm{E}_{\mathrm{soP}}\right)$ and they forms the set EEWE $=\{34,38,44,50\}$ and these elements are not found from theorem3.1 for $n=5$. [There may exist some even weighted edges in the graph BKSTPVEEWG $\left(\mathrm{V}_{\mathrm{atP}}, \mathrm{E}_{\mathrm{soP}}\right)$ which are also existed in the theorem 3.1. except the elements of the set EEWE.]

Again if we consider the graph CPVEEWG $\left(\mathbf{V}_{\mathbf{a t P}}, \mathbf{E}_{\mathbf{s o P}}\right)$ for $n=6$ consecutive prime vertices [theorem3.1] and construct the graph BKSTPVEEWG $\left(\mathrm{V}_{\mathrm{atP}}, \mathrm{E}_{\mathrm{soP}}\right)$, for $\mathrm{n}=7$ consecutive primes vertices then from the graph of figure7 we have 26 even weighted edges five of them are repeated even weights and one three times occurrence even weight 60 and they are 30,32,34, 36,36, 38,40, $\underline{42,42}, 44, \underline{46,46}, \underline{48,48}, 50,52, \underline{54,54}, 56,58, \underline{60,60,60}, 62,66$ and 68.

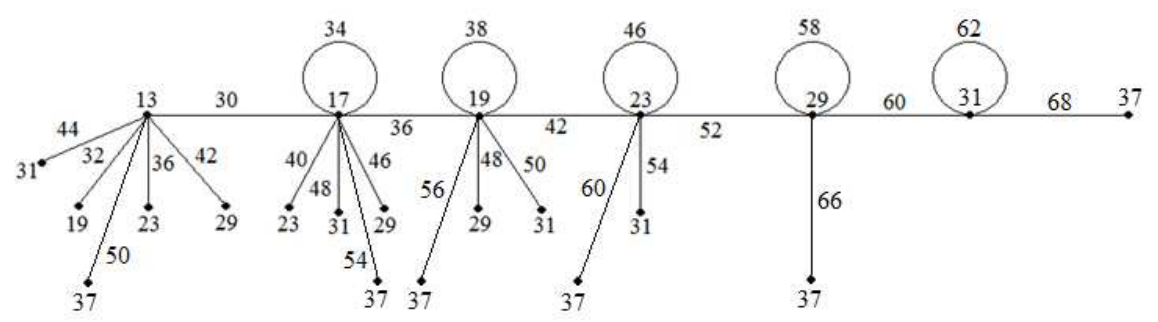

Figure 7

Here we find that the even weighted edges $34,38,56$ and 58 are found from the graph [figure-7] and they again form the set $\mathbf{E E W E}=\{34,38,56,58\}$ and these elements are not found in theorem3.1 for $n=6$.

Similarly, if we consider the graph CPVEEWG $\left(\mathbf{V}_{\mathbf{a t P}}, \mathbf{E}_{\mathbf{s o P}}\right) \mathrm{n}=7$ consecutive primes vertices and construct the graph BKSTPVEEWG $\left(\mathrm{V}_{\mathrm{atP},} \mathrm{E}_{\mathrm{soP}}\right)$ for $n=8$ consecutive prime vertices, then we have the graph of figure- 8 with 34 even weighted edges with six repeated even weights and two three time occurrence even weighted edges 54 and 60 and they are 30,32,34, 36,36, 38,40, 42,42, $44,46,46,48,48,50,50,52,54,54,54,56,58,58,60,60,60,6 \overline{2,64}, 66,68,70, \overline{72,74}$ and 78 .

Here again the even weighted edges $34,38,58,62,64$ [figure-8] form the set EEWE which are not found in theorem 3.1 for $n=7$

Thus, continuing the process of construction of the graph CPVEEWG $\left(\mathbf{V}_{\text {atP }}, \mathbf{E}_{\mathbf{s o P}}\right)$ for $\mathrm{n}=8,9,10 \ldots$ consecutive prime vertices for the complete graph $\mathrm{G}(\mathrm{V}, \mathrm{E})$ and accordingly if we construct the graph BKSTPVEEWG $\left(\mathbf{V}_{\text {atP }}, \mathbf{E}_{\mathbf{s o}} \mathbf{P}\right.$ ) for the prime vertices $\mathrm{P}_{1}<\mathrm{P}_{2}<\mathrm{P}_{3}<\mathrm{P}_{4}<\mathrm{P}_{5} \ldots<\mathrm{P}_{\mathrm{n}}$, for $\mathrm{n}=9,10,11, \ldots$ consecutive prime vertices we have the general pattern of the graph BKSTPVEEWG 


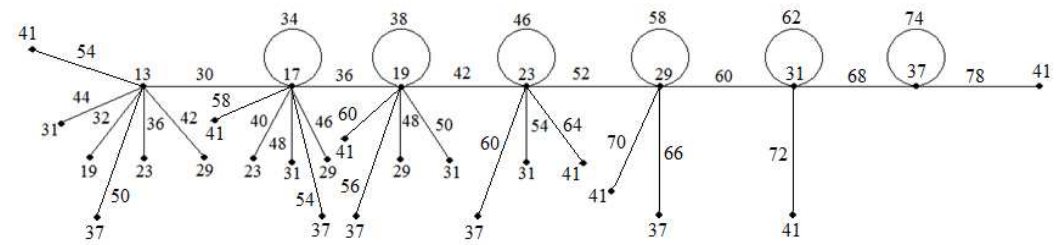

Figure 8

$\left(\mathrm{V}_{\mathrm{atP}}, \mathrm{E}_{\mathrm{soP}}\right)$ which is shown in figure -9, from where all the exceptional even weighted edges can be found from the set EEWE which are not found in the theorem3.1

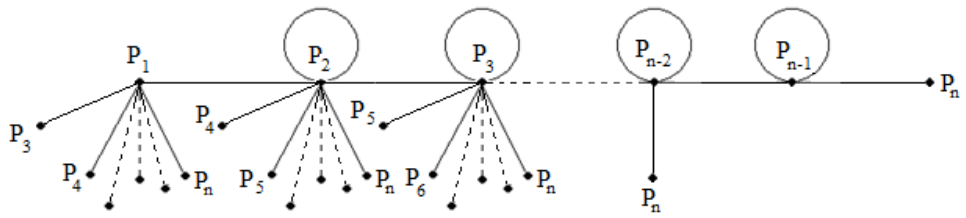

Figure 9

Thus, it is clear that the exceptional even weighted edges which are not obtained in the theorem3.1 can be found from the set EEWE which are found actually from graph BKSTPVEEWG $\left(\mathrm{V}_{\mathrm{atP}}, \mathrm{E}_{\mathrm{soP}}\right)$. The graph of figures 6 , 7,8 and 9 clears the idea of existence of even weighted edges of the graph BKSTPVEEWG $\left(\mathrm{V}_{\mathrm{atP}}, \mathrm{E}_{\mathrm{soP}}\right)$ which can be shown from the graph of figure 10 , where the values of $\mathrm{x}$-axis represents the consecutive prime vertices and the values $\mathrm{y}$-axis represents the even weighted edges that is even numbers.

Hence it is clear from the graphs of Figure-10, that the even weighted edges of the graph BKSTPVEEWG $\left(\mathrm{V}_{\mathrm{atP}}, \mathrm{E}_{\mathrm{so}} \mathrm{P}\right)$ can be found for different values of $\mathrm{n} \geq 6$ consecutive prime vertices from where the different types of the set $\mathbf{E E W E}$ can be found easily for $\mathrm{n} \geq 6$ consecutive primes. The graph of figure-10 gives $19,26,34,43,53,64,76$ and 88 even weighted edges=even numbers for the eight consecutive primes numbers separately. Hence the proof.

Remark 3. In the procedure of the above proof of the theorem 3.2, one may consider the values of primes $\mathrm{n} \geq 5$ as a pair that is one may consider the graph $\mathbf{C P V E E W G}\left(\mathbf{V}_{\mathbf{a t P}}, \mathbf{E}_{\mathbf{s o P}}\right)$ for the values $\mathrm{n}=5,6$ consecutive prime vertices together for two separate graph CPVEEWG $\left(\mathbf{V}_{\text {atP }}, \mathbf{E}_{\mathbf{s o}}\right)$ and then can find the graph BKSTPVEEWG $\left(\mathrm{V}_{\text {atP }}, \mathrm{E}_{\mathrm{soP}}\right)$ for $\mathrm{n}=6,7$ consecutive prime vertices together for two separate graphs etc, which will gives two figures for 


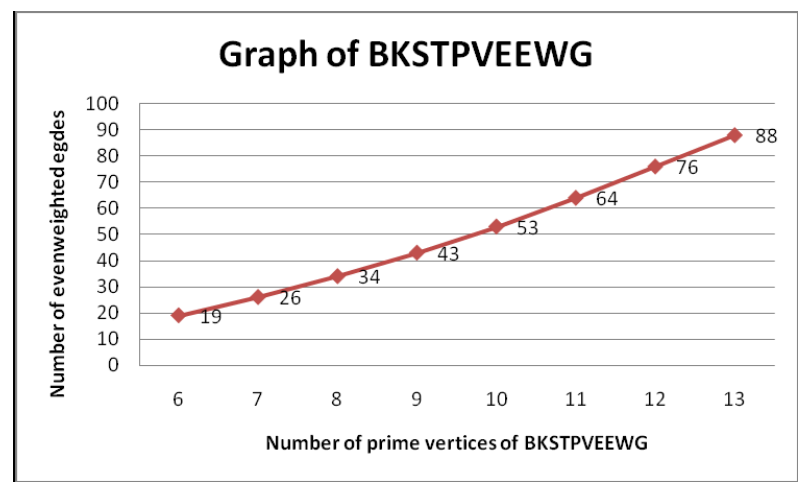

Figure 10

each pair for both the graphs etc and then it will take many figures for each pair of numbers $\geq 8$.

Theorem 3.3. All consecutive even weighted edges $=$ consecutive even numbers $\geq 30$ can be obtained from union of the even weighted edges of the graph CPVEEWG ( $\left.\mathbf{V}_{\mathbf{a t P}}, \mathbf{E}_{\mathbf{s o P}}\right)$ and the elements of the set EEWE.

Proof. It is found from theorem 3.1 that there are $n(n-1) / 2$ number of even weighted edges for $\mathrm{n} \geq 5$ consecutive prime vertices $\mathrm{P}_{1}<\mathrm{P}_{2}<\mathrm{P}_{3}<\mathrm{P}_{4} \ldots<\mathrm{P}_{n}$ which are not consecutive even weighted edges. Again from the theorem 3.2, we have the set EEWE with exceptional even weighted edges which are not found from the theorem 3.1. Now if we take the union of all even weighted edges n(n-1)/2 obtained from theorem 3.1 and the exceptional even weighted edges obtained from theorem 3.2 that is the union of the set EEWE then it gives all consecutive even weighted edges =even numbers. For example, if we take the union the even weighted edges $30,32,36,36,40,42,42,46,48,52$ obtained from the graph CPVEEWG ( $\left.\mathbf{V}_{\mathbf{a t P}}, \mathbf{E}_{\mathbf{s o P}}\right)$ [ figure-4] for $\mathrm{n}=5$, for five consecutive prime vertices $13,17,19,23$ and 29 and with the set $\mathbf{E E W E}=\{34,38,44,50\}$ which is obtained from the graph BKSTPVEEWG $\left(\mathrm{V}_{\mathrm{atP}}, \mathrm{E}_{\mathrm{soP}}\right)$ for $\mathrm{n}=6$, consecutive prime vertices, we have a set of 12 consecutive even numbers =even weighted edges which is $\{30,32,34,36,38,40,42,44,46,48,50,52\}$.

Similarly if we take the union of the even weighted edges $30,32,36,42,44,48$, 46,40,36,50,48,42,54,52 and 60 which are obtained from the graph CPVEEWG $\left(\mathbf{V}_{\text {atP }}, \mathbf{E}_{\mathbf{s o P}}\right)$ for $\mathrm{n}=6$ consecutive prime vertices with the set $\mathbf{E E W E}=\{34,38,56$, $58\}$ which is obtained from the graph BKSTPVEEWG $\left(V_{\text {atP }}, E_{\text {soP }}\right)$ for $n=7$ consecutive prime vertices, we have a set of 16 consecutive even numbers which is 
$\{30,32,34,36,38,40,42,44,46,48,50,52,54,56,58,60\}$.

Again if we take the union of the even weighted edges $30,32,36,42,44,50,36,40$, $46,48,54,42,48,50,56,52,54,60,60,66,68$ which are obtained from the graph CPVEEWG $\left(\mathbf{V}_{\mathbf{a t P}}, \mathbf{E}_{\mathbf{S o P}}\right)$ for the $n=7$ consecutive prime vertices with the set $\mathbf{E E W E}=\{34,38,58,62,64\}$ which is obtained from the graph BKSTPVEEWG $\left(\mathrm{V}_{\text {atP }}, \mathrm{E}_{\mathrm{soP}}\right)$ for $\mathrm{n}=8$ consecutive prime vertices, we have a set of 20 consecutive even numbers which is $\{30,32,34,36,38,40,42,44,46,48,50,52,54,56,58,60,60,64,66$, $68\}$.

Continuing the process of taking the union of the even weighted edges of the graph CPVEEWG $\left(\mathbf{V}_{\mathbf{a t P}}, \mathbf{E}_{\mathbf{s o P}}\right)$ for $\mathrm{n}=8,9,10,11 \ldots$ Consecutive prime vertices with the sets EEWE, which are obtained from the graph BKSTPVEEWG $\left(\mathrm{V}_{\mathrm{atP}}, \mathrm{E}_{\mathrm{soP}}\right)$ for $\mathrm{n}=9,10,11,12 \ldots$ Consecutive prime vertices, we have different type of sets of 25 consecutive even numbers, 28 consecutive even numbers, 31 consecutive even numbers, 36 consecutive even numbers. Finally we see that the union of the above different even weighted edges obtained from theorem3.1 and the different type of sets EEWE of even weighted edges obtained from the theorem 3.2 gives consecutive even weighted edges which are actually consecutive even numbers $\geq 30$. This completes the proof.

\section{Proof of Gold Bach Conjecture}

Statement. Every even number $n>2$ can be expressed as a sum of two primes.

Proof. Proof is very simple, which follows from the theorem 3.3. From the theorem 3.3 we have the consecutive even weights $\mathrm{w}_{1}, \mathrm{w}_{2}, \mathrm{w}_{3}, \mathrm{w}_{4}, \mathrm{w}_{5} \ldots \mathrm{w}_{\infty}$ which are obtained as a sum of prime vertices $V_{1}, V_{2}, V_{3}, \ldots$ for the primes $\mathrm{P}_{1}, \mathrm{P}_{2}, \mathrm{P}_{3} \ldots$ from the graphs CPVEEWG $\left(\mathbf{V}_{\mathbf{a t P}}, \mathbf{E}_{\mathbf{s o P}}\right)$ and from the graph BKSTPVEEWG $\left(\mathrm{V}_{\mathrm{atP}}, \mathrm{E}_{\mathrm{soP}}\right)$ from where the set of edges EEWE is obtained and their union gives the infinite number of even numbers. This immediately follows that the Goldbach conjecture is true for the even numbers from 2 to $(10)^{\infty}$, as the consecutive even weighted edges are the consecutive even numbers $\geq 30$ which are actually obtained as a sum of two prime weights=prime numbers. [experimentally, the Gold Bach conjecture has been shown for the even numbers 2 to $(10)^{18}[5]$. Hence every even number $n>2$ can be expressed as a sum of two primes, where the sum of two prime give the weighted edge of the graph.

This completes the proof of the conjecture.

Remark 3. The Goldbach conjecture is true for all even numbers which are obtained from the weighted edges of the two graphs $\mathbf{C P V E E W G}\left(\mathbf{V}_{\mathbf{a t P}}, \mathbf{E}_{\mathbf{s o P}}\right)$ 
and the graph BKSTPVEEWG $\left(\mathrm{V}_{\text {atP }}, \mathrm{E}_{\mathrm{soP}}\right)$. Hence it is not big problems in number theory, which can be proved easily from graph theory

\section{Conclusion}

We have supplied the proof of long standing Gold Bach conjecture with the some graph theoretic concept considering the weighted edges as an even number which are obtained as a sum of two prime weights=prime numbers.

\section{References}

[1] G.P. Loweke, The Lour of Prime Numbers, Vontage press, N.Y. (1982).

[2] Richard Courant, H. Robbins, What is Mathematics, Oxford University Press (1996).

[3] B. Kalita, Some conjectural problems of primes and partial solution of it, In: International Conferences on Recent Advances in Mathematical Sciences, I.I.T. Kharagpur (2000).

[4] B. Kalita, Proof of goldbach conjecture and related conjecture of primes, Bull. Pure and Applied Sciences, 25E, No. 2 (2006), 451-459.

[5] V. Alex, et al, Goldbach suspects a step further, Sources Kennislink, Language, Dutch, On-line article (2007).

[6] B. Kalita, Certain forms of even numbers and their properties, Global Journal of Pure and Applied Mathematics, 5, No. 1 (2009), 53-58.

[7] B. Kalita, et al, Some properties of graphical partitions, Int. Journal of Appl. Engineering Research, 4, No. 10 (2009), 1851-1856.

[8] C. Pomerance, The Search for Prime Numbers, Scientific American (1982).

[9] P. Erdos, H.L. Montgomery, Sum of numbers with many divisors, J. Number Theory, 75, No. 1 (1999). 1-6.

[10] H. Yokota, The largest integer expressible as a sum of reciprocal of integers, J. Num. Thory, 76, No. 2 (1999). 
[11] C.C. Rousseau, F. Ali, On a conjecture concerning graphical partitions congruent, Number 104 (1994), 150-160; A note on graphical partitions, $J$. Com. Th. Series B, 64 (1995), 314-318.

[12] M. Jennifer, et al, Graphical basis partitions, Graph and Comb., 4 (1998), $241-261$.

[13] F. Harary, Graph Theory, Narosa Publishing House,

[14] B. Kalita, A new set of non-planar graphs, Bull. Pure and Applied Sci., 24E, No. 1 (2005), 29-38.

[15] B. Kalita, Some Investigations on Graph Theory, Ph.D. Thesis, Finance India, XIX, No. 4 (2005), 1430-1438.

[16] B. Kalita, Sub-graphs of complete graphs, In: Proc. International Conference on Foundations of Computer Sci., Las Vegas, USA (2006), 71-77.

[17] B. Douglas, Introduction to Graph Theory, Pearson Education (2002), 260269. 